\title{
Acute Myocarditis in a Patient Following mRNA-1273 SARS-CoV-2 Vaccination
}

\author{
Toshinaru Kawakami, Kazuyuki Yahagi, Masahiro Sekiguchi, Taiki Ishizawa, \\ Hideaki Nonaka, Naoto Setoguchi, Yusuke Watanabe, Masaaki Nakase, Yu Horiuchi, \\ Masahiko Asami, Hitomi Yuzawa, Kota Komiyama, Jun Tanaka, Jiro Aoki and Kengo Tanabe
}

\begin{abstract}
:
We herein report a case of acute myocarditis possibly related to the second dose of an mRNA-COVID-19 vaccine in a 45-year-old woman with no remarkable medical history. She had a fever for one week following the second dose of the mRNA-1273 SARS-CoV-2 vaccine. One week later, she presented with chest pain and electrocardiogram changes. Her serum troponin levels were elevated upon admission. Echocardiography showed segmental wall motion abnormalities of the apex, apical portion of the anterior and inferior walls. The findings of cardiac magnetic resonance imaging were consistent with acute myocarditis.
\end{abstract}

Key words: Acute myocarditis, SARS-CoV-2 vaccination

(Intern Med Advance Publication)

(DOI: 10.2169/internalmedicine.9000-21)

\section{Introduction}

Coronavirus disease 2019 (COVID-19) is spreading worldwide, and the number of infected people is increasing. Simultaneously, COVID-19 vaccines, particularly mRNA vaccines, have been developed, but adverse events associated with these vaccines are also being reported increasingly frequently worldwide (1).

Acute myocarditis and pericarditis are considered rare complications following COVID-19 vaccination, especially in young adults and adolescent males (1). Most cases of post-vaccination myocarditis show a disease onset within approximately four days after the second dose of the COVID19 vaccine (1).

We herein report a case of acute myocarditis, occurring two weeks after the second dose of the mRNA-COVID-19 vaccine with multiple imaging modalities in a Japanese patient.

\section{Case}

A 45-year-old woman with no remarkable medical history presented with chest pain following the second dose of the mRNA-1273 SARS-CoV-2 vaccine (Moderna, Cambridge, MA, USA) received 2 weeks earlier. Of note, the patient had a fever $\left(38-40{ }^{\circ} \mathrm{C}\right)$ for 1 week after receiving the second dose of the vaccine, which had been given 28 days after the first dose. Nasopharyngeal swab testing for SARS-CoV-2 prior to admission was negative.

Vital signs on admission were as follows: temperature; $37.3{ }^{\circ} \mathrm{C}$, blood pressure; $138 / 99 \mathrm{mmHg}$, heart rate; 90 beats per minute, and oxygen saturation; $96 \%$ in room air. A physical examination was unremarkable, and heart sounds were regular and without any murmur or friction. Laboratory data revealed elevated levels of cardiac troponin-T (100 ng/L), NT-pro BNP (1,930 pg/mL), and C-reactive protein $(1.78 \mathrm{mg} / \mathrm{dL})$. Creatinine kinase and creatinine kinase-MB levels were within the normal ranges. White blood cells were $9,400 / \mu \mathrm{L}$ with a normal range of eosinophils.

An electrocardiogram (ECG) showed an inverted $\mathrm{T}$ wave in the inferior leads (II, III, and aVF) (Fig. 1). Chest X-ray showed mild pulmonary edema. Transthoracic echocardiography (TTE) showed a mildly reduced ejection fraction with segmental wall motion abnormalities of the apex and inferior and lateral wall. Coronary angiography on the day

Division of Cardiology, Mitsui Memorial Hospital, Japan

Received: November 12, 2021; Accepted: January 20, 2022; Advance Publication by J-STAGE: March 5, 2022

Correspondence to Dr. Kazuyuki Yahagi, yyahakazu@gmail.com 


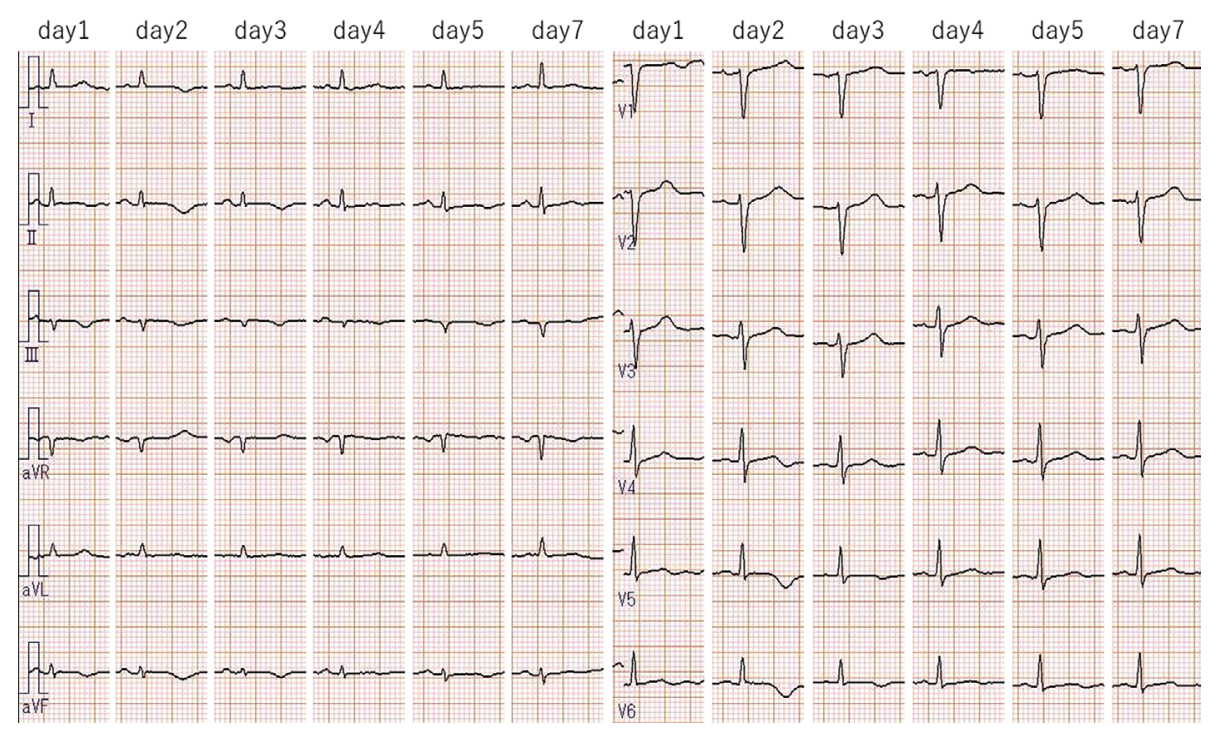

Figure 1. An electrocardiogram at admission showed T-wave inversion in the inferior leads (II, III, aVF). T-wave inversion occurred in V5 and V6 on day 2. T-wave inversion normalized on day 7.
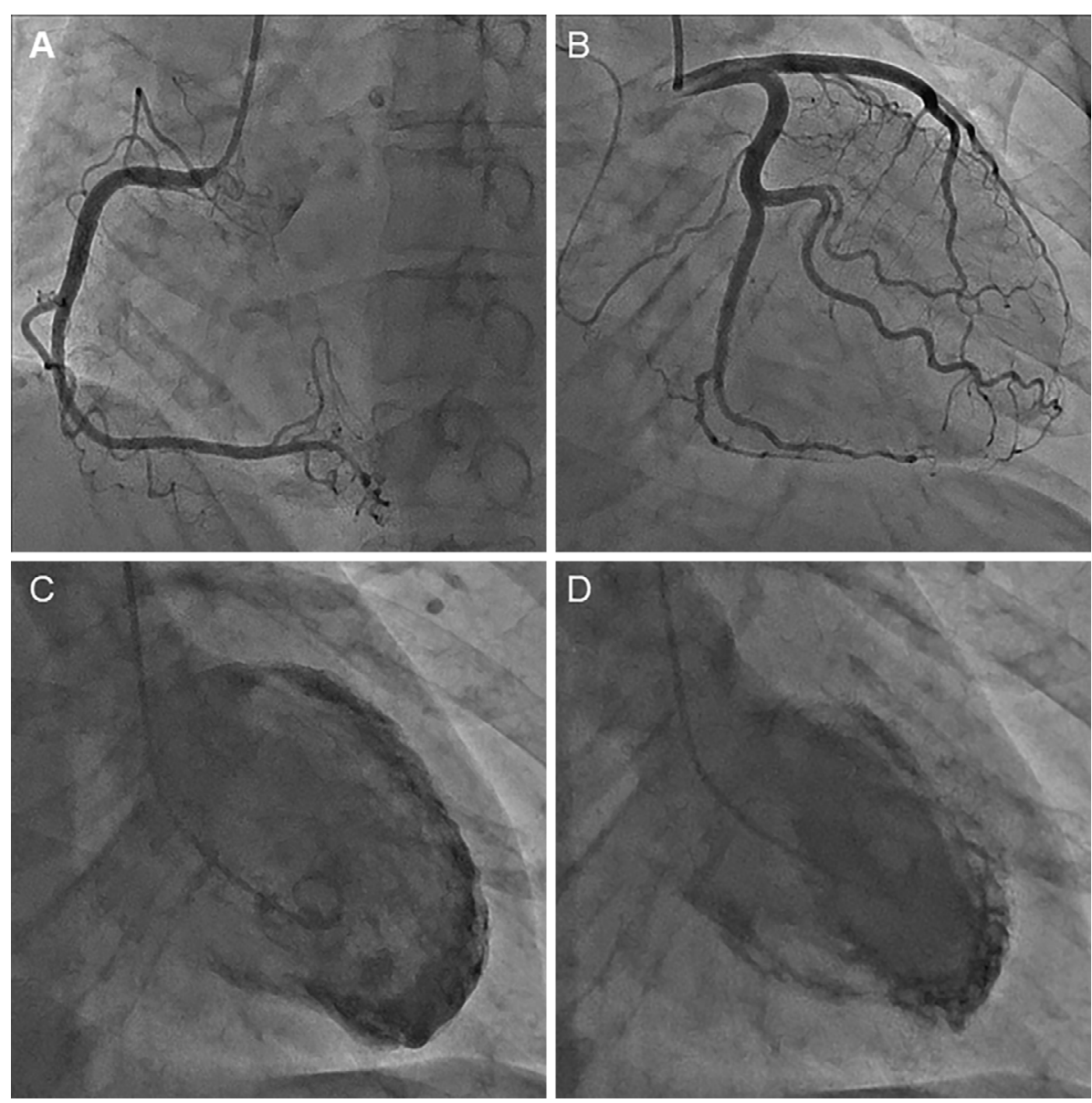

Figure 2. Coronary angiography of the right coronary artery (A) and the left coronary artery (B). Left ventriculography at the end-diastolic phase (C) and the end-systolic phase (D).

of admission revealed a normal coronary artery. Left ventriculography showed decreased wall motion in the apex and inferior and lateral wall (Fig. 2). Cardiac magnetic resonance imaging (MRI) on day two showed increased myocardial signal intensity (edema) of the apex and inferior and lateral wall in T2-weighted images. Late gadolinium enhancement (LGE) imaging demonstrated diffuse hyperenhancement, especially in the apex and inferior and lateral wall. Native T1mapping showed corresponding abnormalities with elevated values in the apex and inferior and lateral wall (Fig. 3). The 

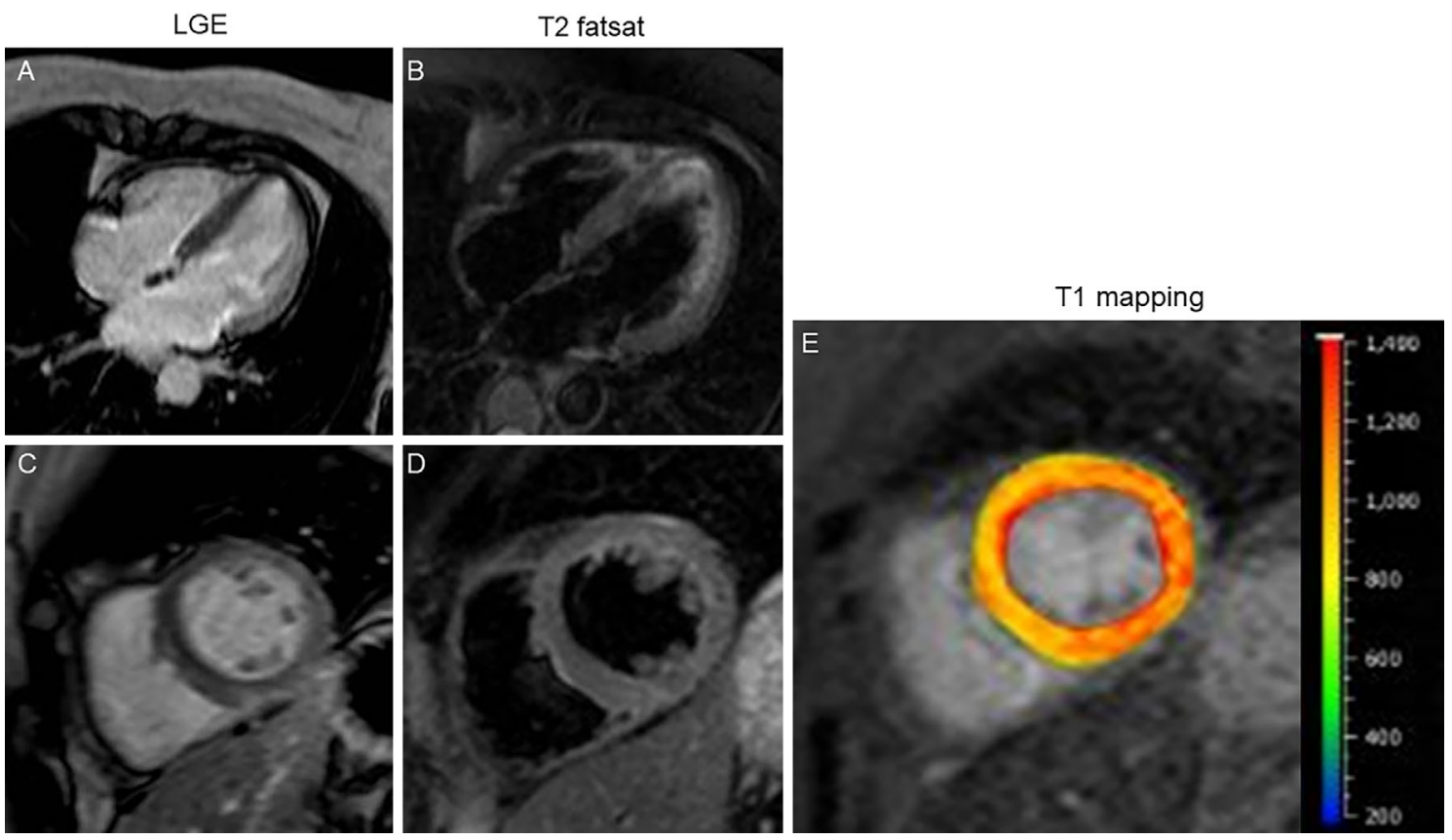

Figure 3. Late gadolinium enhancement imaging in the four-chamber long axis (A) and the short axis (B). T2-weighted image in the four-chamber long axis (C) and the short axis (D). Native T1mapping in the short axis (E).

cardiac MRI findings were compatible with acute myocarditis based on the revised Lake Louise criteria (2). Based on these findings, we diagnosed the patient with acute myocarditis.

Extensive infectious and rheumatologic examinations were unremarkable. A quantitative SARS-CoV-2 antibody assay (Abbott Diagnostics, Chicago, IL, USA) was performed on day two, showing a high titer $(290,022 \mathrm{AU} / \mathrm{m})$. The patient had spontaneous remission, and her chest discomfort regressed quickly. With regard to the ECG, the inverted T wave in the inferior leads became normal on day four. Follow-up TTE on day five demonstrated improved wall motion of the inferior wall, apex, and lateral wall. Troponin$\mathrm{T}$ returned to normal levels on day seven, and the patient was discharged on day eight.

\section{Discussion}

The most common cause of acute myocarditis is viral infection. The most frequently implicated viruses in North America and Europe are the enteroviruses, including coxsackievirus. Parvovirus B-19 and human herpesvirus 6 are frequent culprits (3). Post-vaccination myocarditis has also been reported, especially after smallpox vaccination (4).

Recently, myocarditis has been recognized as a complication of COVID-19 vaccination. Previous reports of myocarditis after COVID-19 vaccination have shown that most cases occur in young adult and adolescent men, usually two to three days after the second dose of vaccination (1). A report from Israel reported 136 cases of myocarditis after COVID-19 vaccination (5). Of the 136 cases, 117 presented after the second dose. The time to the onset of the disease varied from 1 to 30 days after vaccination, and the number peaked at three days after vaccination. However, myocarditis after COVID-19 vaccination is extremely rare, and the cause of myocarditis remains unknown.

The diagnosis of acute myocarditis can be confirmed by cardiac MRI, and it is a useful tool for monitoring the disease activity (6). The diagnostic criteria for myocarditis, the Lake Louise Criteria, were developed in 2009 and updated in 2018 (2). In the current case, cardiac MRI without contrast was performed at day 10 , and the findings did not change significantly. These findings are compatible with a previous report, which stated that myocardial edema of acute myocarditis normalized after four to eight weeks of follow-up (6).

With regard to the treatment, according to a previous report (7), some patients received one or a combination of anti-inflammatory drugs, such as non-steroidal antiinflammatory drugs, colchicine, and prednisone. Other patients received no medication. All of them were hemodynamically stable and did not require either inotropes or mechanical support. As our patient was hemodynamically stable and showed quick relief of symptoms with normalization of laboratory data, ECG, and TTE, we decided to perform follow-up and monitor her closely with no medication.

Although the exact mechanism underlying postvaccination myocarditis is unknown, evidence suggests that molecular mimicry plays a role (8). Molecular mimicry is one of the leading mechanisms by which infectious agents induce autoimmunity. It occurs when similarities between foreign and self-peptides favor the activation of autoreactive $\mathrm{T}$ or $\mathrm{B}$ cells by a foreign-derived antigen in a susceptible individual (9). A previous report described two cases of his- 
tologically confirmed myocarditis after COVID-19 mRNA vaccination (10). Based on left ventriculography findings, takotsubo syndrome was considered as a differential diagnosis. Although an endomyocardial biopsy is important to prove the cause of myocarditis histologically, we decided not to risk an endocardial biopsy for safety reasons. Similar to our case, in most cases of myocarditis after COVID-19 vaccination, myocarditis occurs after the second shot, probably due to a stronger immune response than with the first dose. One report suggested that a high post-vaccination titer was probably associated with the risk of developing myocarditis after COVID-19 vaccination (8). A higher postvaccination titer presumably indicates a stronger immune response, thereby causing the immune system to misfire.

Although myocarditis after COVID-19 vaccination is rare in most patients, rapid recovery and screening by cardiac markers, ECG, TTE, and cardiac MRI are important for the early diagnosis in patients complaining of chest discomfort after COVID-19 vaccination.

\section{Conclusion}

We encountered a 45-year-old woman who developed chest pain after receiving the second dose of the mRNA1273 COVID-19 vaccine and who was diagnosed with acute myocarditis. Overall, vaccination-related myocarditis is a rare and mostly mild adverse event. As in this case, cardiac MRI seems to be a useful tool for making a diagnosis. Further research will be needed to confirm the association of myocarditis with COVID-19 vaccination.

The authors state that they have no Conflict of Interest (COI).

\section{Disclosure}

The authors have no conflicts of interest to disclose.

\section{References}

1. Rosner CM, Genovese L, Tehrani BN, et al. Myocarditis Temporally Associated With COVID-19 Vaccination. Circulation 144: 502-505, 2021

2. Ferreira VM, Schulz-Menger J, Holmvang G, et al. Cardiovascular Magnetic Resonance in Nonischemic Myocardial Inflammation. J Am Coll Cardiol 72: 3158-3176, 2018.

3. Kang M, An J. Viral Myocarditis. 2021 Aug 11. In: StatPearls [Internet]. Treasure Island (FL): StatPearls Publishing [cited 2021 Jan -].

4. Su JR, McNeil MM, Welsh KJ, et al. Myopericarditis after vaccination, Vaccine Adverse Event Reporting System (VAERS), 19902018. Vaccine 39: 839-845, 2021.

5. Mevorach D, Anis E, Cedar N, et al. Myocarditis after BNT162b2 mRNA Vaccine against Covid-19 in Israel. N Eng J Med 385: 2140-2149, 2021.

6. Luetkens JA, Homsi R, Dabir D, et al. Comprehensive Cardiac Magnetic Resonance for Short-Term Follow-Up in Acute Myocarditis. J Am Heart Assoc 5 (7): e003603, 2016.

7. Larson KF, Ammirati E, Adler ED, et al. Myocarditis After BNT 162b2 and mRNA-1273 Vaccination. Circulation 144: 506-508, 2021.

8. D'Angelo T, Cattafi A, Carerj ML, et al. Myocarditis After SARSCoV-2 Vaccination: A Vaccine-Induced Reaction? Can J Cardiol 2021S0828-282X(21)00286-5.

9. Rojas M, Restrepo-Jiménez P, Monsalve DM, et al. Molecular mimicry and autoimmunity. J Autoimmun 95: 100-123, 2018.

10. Verma AK, Lavine KJ, Lin CY. Myocarditis after Covid-19 mRNA Vaccination. N Engl J Med 385: 1332-1334, 2021.

The Internal Medicine is an Open Access journal distributed under the Creative Commons Attribution-NonCommercial-NoDerivatives 4.0 International License. To view the details of this license, please visit (https://creativecommons.org/licenses/ by-nc-nd/4.0/).

(C) The Japanese Society of Internal Medicine Intern Med Advance Publication 